\title{
E-consults: an effective way to decrease clinic wait times in rheumatology
}

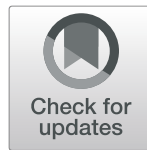

Veena Patel ${ }^{1^{*}}$ (D), Diana Stewart ${ }^{2}$ (D) and Molly J. Horstman ${ }^{3,4,5}$ (D)

\begin{abstract}
Background: To evaluate the effect of E-consults on wait times and resource utilization for positive antinuclear antibody (ANA) referrals in outpatient rheumatology.

Methods: We conducted a pre-post study of E-consult implementation for positive ANA referrals. We retrospectively reviewed "positive ANA" referrals from 1/2015-3/2017. A statistical process control chart was created to display monthly average wait times for in-person clinic visits and to identify special cause variation. Final diagnoses, wait times and resource utilization were recorded and compared between E-consults and in-person referrals.

Results: There were 139 referrals for positive ANA with 126 occurring after E-consult implementation in August 2015. Forty-four percent (55/126) of referrals were E-consults; $76 \%$ did not have an in-person visit after initial electronic rheumatology recommendation. A control chart demonstrated special cause variation in the form of a shift from June 2016 - January 2017, suggesting a temporal association between decreased wait times and the implementation of E-consults. Eleven patients were diagnosed with ANA-associated rheumatic disease; the majority of patients $(73 \%, 86 / 139)$ did not have a rheumatologic diagnosis. Overall E-consults utilized more labs than inperson visits, but this was not statistically significant. In-person visits utilized more imaging studies, which was statistically significant.
\end{abstract}

Conclusion: E-consults are an effective way to address positive ANA consults without significant increase in resource utilization and were temporally associated with decreased wait times for in-person visits.

Keywords: Quality improvement, Telemedicine, Referral and consultation, Rheumatology

\section{Background}

The term telehealth refers to multiple modalities to provide care remotely using some form of technology. Due to the COVID-19 pandemic, there has been a rapid increase in telehealth usage in 2020 [1]. One type of telehealth format, the electronic consult (E-consult), has been utilized by healthcare systems in the United States and internationally for many years $[2,3]$ as a way to help patients gain access to specialty care. E-consults are an

\footnotetext{
* Correspondence: Veena.patel@austin.utexas.edu

'Division of Rheumatology, Department of Medicine, University of Texas at Austin, Dell Medical School, 1601 Trinity St., Bldg B, Stop Z0900, Austin, TX 78712, USA

Full list of author information is available at the end of the article
}

asynchronous form of communication between referring provider and specialist through a shared EHR (electronic health record) or web-based platform that entails the specialist reviewing chart data and replying with recommendations electronically $[1,3]$. E-consults have facilitated a decrease in face-to-face visits, increased access to care, and improved provider satisfaction [3]. E-consults also affected wait times, from shortening clinic wait times or the perception of wait times, but multiple contributing factors in real-world settings have made it difficult to precisely assess this impact [3, 4].

Prior studies have shown the benefit of synchronous telehealth modalities within rheumatology such as videoconference visits [5-9], but there is little published about

(c) The Author(s). 2020 Open Access This article is licensed under a Creative Commons Attribution 4.0 International License, which permits use, sharing, adaptation, distribution and reproduction in any medium or format, as long as you give appropriate credit to the original author(s) and the source, provide a link to the Creative Commons licence, and indicate if changes were made. The images or other third party material in this article are included in the article's Creative Commons licence, unless indicated otherwise in a credit line to the material. If material is not included in the article's Creative Commons licence and your intended use is not permitted by statutory regulation or exceeds the permitted use, you will need to obtain permission directly from the copyright holder. To view a copy of this licence, visit http://creativecommons.org/licenses/by/4.0/. The Creative Commons Public Domain Dedication waiver (http://creativecommons.org/publicdomain/zero/1.0/) applies to the data made available in this article, unless otherwise stated in a credit line to the data. 
the impact of asynchronous E-consults. Scheibe et al. [10], reviewed the impact of another type of asynchronous telehealth format, the pre-consult exchange, which uses a similar strategy as E-consults, but with more integrated communication between the referring provider and specialist. One study evaluated E-consults in rheumatology and showed advantages including quicker turn-around times, a decrease in face-to-face visits, and increased referring provider satisfaction. Common reasons E-consults were utilized in this study included treatment questions, questions about whether a patient required an in-person evaluation, and interpretation of a positive laboratory test [2].

A positive antinuclear antibody (ANA) test is a common reason for referral to rheumatology. The ANA is a nonspecific test and is often checked in the absence of clinical signs and symptoms of an ANA-associated rheumatic disease (AARD). Studies have shown that a positive ANA resulting in a diagnosis of an AARD is low (11\% or less) $[11,12]$. Another study reported when an ANA was checked without using proper clinical criteria, $88 \%$ of positive cases had no systemic rheumatic disease diagnosis [13]. ANA positivity is present in the general healthy population [14, 15], and its prevalence has increased in the past 25 years [16]. Given the increase in prevalence in healthy individuals coupled with the shortage of rheumatologists in the US [17] sending referrals for positive ANA is an inefficient use of limited resources.

Finding ways to address positive ANA referrals promptly, such as through the use of E-consults, can improve access for patients requiring in-person rheumatology evaluation. This project aims to evaluate the resource utilization of Econsults and their effect on wait times for positive ANA referrals in a rheumatology clinic.

\section{Methods}

We conducted a pre-post study of E-consult implementation for positive ANA referrals. Our outcomes included wait times, diagnostic tests ordered, and patients' final diagnoses.

The Michael E. DeBakey VA Medical Center is a tertiary health care center consisting of inpatient and outpatient medical services caring for 113,000 Veterans in Southeast Texas. An EHR is used by all providers throughout this system and any provider within the system can order a referral for specialty care. The referring provider places an order for a referral to rheumatology in the EHR; reason for consult is entered into a free text box within the referral order. Starting in August 2015, the referring provider had the option of selecting either "E-consult" or "in-person visit" when placing a referral to rheumatology.

The referral review process was completed by one faculty rheumatologist who reviewed all referral requests and determined if the type of referral selected by the referring clinician (in-person visit or E-consult) was appropriate. The reviewer had the option to switch the type of consult if needed. For E-consults, the reviewer electronically replied through the EHR to the referring clinician with recommendations after reviewing the patient's electronic chart. Rheumatology staff scheduled clinic appointments for in-person referrals.

We retrospectively reviewed positive ANA referrals to outpatient rheumatology from January 1, 2015, to March 31, 2017. We defined a positive ANA as a result with a titer greater than or equal to 1:40 (our lab reference range) by indirect immunofluorescence. Through chart review, we collected demographic data, referral information, wait times, labs, and imaging ordered during the first rheumatology evaluation and final diagnoses. Chart review was done using a standard data abstraction form with standard definitions of every type of extracted data and standard locations for identifying this data from the EHR.

We defined wait time for an in-person visit as the time from referral placement to the rheumatology clinic appointment. E-consult wait time was defined as the time from referral placement to the rheumatologist's initial electronic response. We calculated the average monthly wait time for a positive ANA in-person visit and graphed the results using an $\mathrm{XmR}$ statistical process control chart to assess for special cause variation [18]. An unpaired Ttest was used to compare wait times and resource utilization between visit types in total, and for each final diagnosis category.

Resource utilization was defined as rheumatologic labs and imaging ordered during the first rheumatology evaluation. The following labs were included in data collection: rheumatoid factor, anti-cyclic citrullinated peptide (antiCCP), anti-Ro (SS-A), anti-La (SS-B), anti-Smith, antinRNP, anti-double stranded DNA (dsDNA), complement $\mathrm{C} 3$ and $\mathrm{C} 4, \mathrm{Scl}-70$, sedimentation rate, C-reactive protein, antiphospholipid antibody panel, antineutrophilic cytoplasmic antibodies, cryoglobulins, aldolase, HLA-B27, uric acid, and anti-Jo1. Imaging studies recorded included joint X-Rays, body or joint computed tomography (CT) scans, and joint magnetic resonance imaging (MRI) scans. Resource utilization was calculated as the mean per person and for each final diagnosis category.

At the time of review, patients' final diagnoses were recorded from the assessment and plan section of the rheumatologist's most recent clinic note in the EHR. Diagnoses were organized into the following categories: ANAassociated rheumatic disease (AARD), other rheumatic disease (ORD), no rheumatic disease, or no diagnosis at the time of review. AARD included the following diagnoses: systemic lupus erythematosus (SLE), Sjogren's syndrome, scleroderma, mixed connective tissue disease (MCTD), polymyositis $(\mathrm{PM})$, dermatomyositis $(\mathrm{DM})$, undifferentiated 
connective tissue disease (UCTD), and drug-induced lupus [15]. ORD included diseases that would require routine rheumatology follow-up, but in which an ANA is not used for the diagnosis.

This study was reviewed by the Institutional Review Board (IRB) and the local Veterans Affairs Research and Development Committee and designated as a quality improvement project which did not require formal IRB approval.

\section{Results}

There were 139 positive ANA referrals within our system from January 1, 2015, to March 31, 2017. The majority of patients were white $(n=78,56 \%)$ and male $(n=94,68 \%)$, with an average age of 54 years (Table 1 ). Of the 139 positive ANA referrals, 126 occurred after E-consult implementation in August 2015. While referring providers selected the initial consult type, $13 \%$ of in-person referrals (13/97) were switched to E-consults by the reviewing rheumatologist (Fig. 1). No E-consults were initially switched to in-person visits during the study period.

E-consults were utilized in 44\% (55/126) of patients with an average response time of 1.7 days. An in-person visit was deemed unneeded after the initial rheumatologic recommendation for $76 \%(42 / 55)$ of E-consults. During the duration of this study, none of these Econsults were subsequently scheduled for an in-person visit after initial E-consultation.

The average in-person visit wait time for a positive ANA referral decreased from 64 to 34 days after Econsult implementation $(p<0.001)$. Special cause variation [18] was identified as a shift in the control chart from June 2016 to January 2017 (Fig. 2).

Eleven Veterans $(8 \%)$ had a diagnosis of AARD: SLE $(n=5)$, limited systemic sclerosis $(n=1)$, UCTD $(n=1)$,

Table 1 Demographic characteristics of patients

\begin{tabular}{ll}
\hline \multicolumn{2}{l}{ Characteristics of Patients Referred for Positive ANA } \\
\hline Sex & $\mathrm{n}(\%)$ \\
Female & $45(32 \%)$ \\
Male & $94(68 \%)$ \\
Race & \\
White & $78(56 \%)$ \\
Black & $51(37 \%)$ \\
Hispanic & $8(6 \%)$ \\
Al/Hawaiian & 1 \\
Asian & 1 \\
Age & \\
Age Average & 54 \\
Standard Dev & 14 \\
Age Range & $28-89$ \\
\hline
\end{tabular}

MCTD/SLE overlap $(n=1)$, Sjogren's syndrome $(n=1)$, DM $(n=1)$ and drug-induced lupus $(n=1)$. ORDs were diagnosed in 21 Veterans and included: gout, nonradiographic ankylosing spondylitis, fibromyalgia, sarcoidosis, cryoglobulinemia, polymyalgia rheumatica, and rheumatoid arthritis. The majority of referrals resulted in no rheumatic diagnosis $(86 / 139,62 \%)$. Of the 13 referrals that were switched from in-person visits to Econsults by the reviewing rheumatologist, one was ultimately diagnosed with rheumatoid arthritis and secondary Sjogren's syndrome. The E-consult for this patient was used to order more specific lab work before the inperson visit was scheduled.

E-consults ordered more labs overall, but this was not statistically significant. In-person visits ordered more imaging overall, which was statistically significant (Table 2). The most common labs ordered by E-consults were anti-Smith $(n=32)$, anti-Ro/La $(n=31)$, anti-nRNP $(n=$ $31)$, and dsDNA $(n=29)$. The most common labs ordered by in-person visits after the implementation of Econsults were anti-Ro/La $(n=22), \mathrm{C} 3 / 4(n=22)$, dsDNA $(n=21)$, anti-Smith $(n=19)$.

\section{Discussion}

E-consults are an effective way to address ANA referrals quickly and improve access to rheumatology for patients requiring in-person evaluation without a significant increase in resource utilization.

The effect of E-consults on wait times in prior studies have been variable from a decrease in wait times [4] to no change $[2,10]$. In our study, detecting special cause variation through a control chart suggests a temporal association between E-consult introduction and decreased wait times. We know of no changes that occurred in the rheumatology clinic at that time that would otherwise contribute to the decreased wait time. The reduction in wait time for in-person visits has other potential positive downstream effects including improving value for healthcare systems and reducing patient costs by decreasing unnecessary travel and time off work for in-person appointments.

In our study, E-consults utilized more lab tests than in-person visits, but this was not statistically significant. We reported $7 \mathrm{E}$-consults which resulted in a rheumatic diagnosis (3 AARD and 4 ORD) during our study. These cases used the E-consult to initiate lab work-up before an in-person visit was scheduled. These patients were subsequently followed up in clinic. There are certain cases, such as these, when an in-person visit is needed after E-consult and efficiencies are gained by initiating work-up through the E-consult, making the in-person visit more productive. Given the non-specific nature of the indirect immunofluorescence ANA test $[11,15]$, Econsults, for this reason, may also require more specific lab data before warranting an in-person evaluation. 


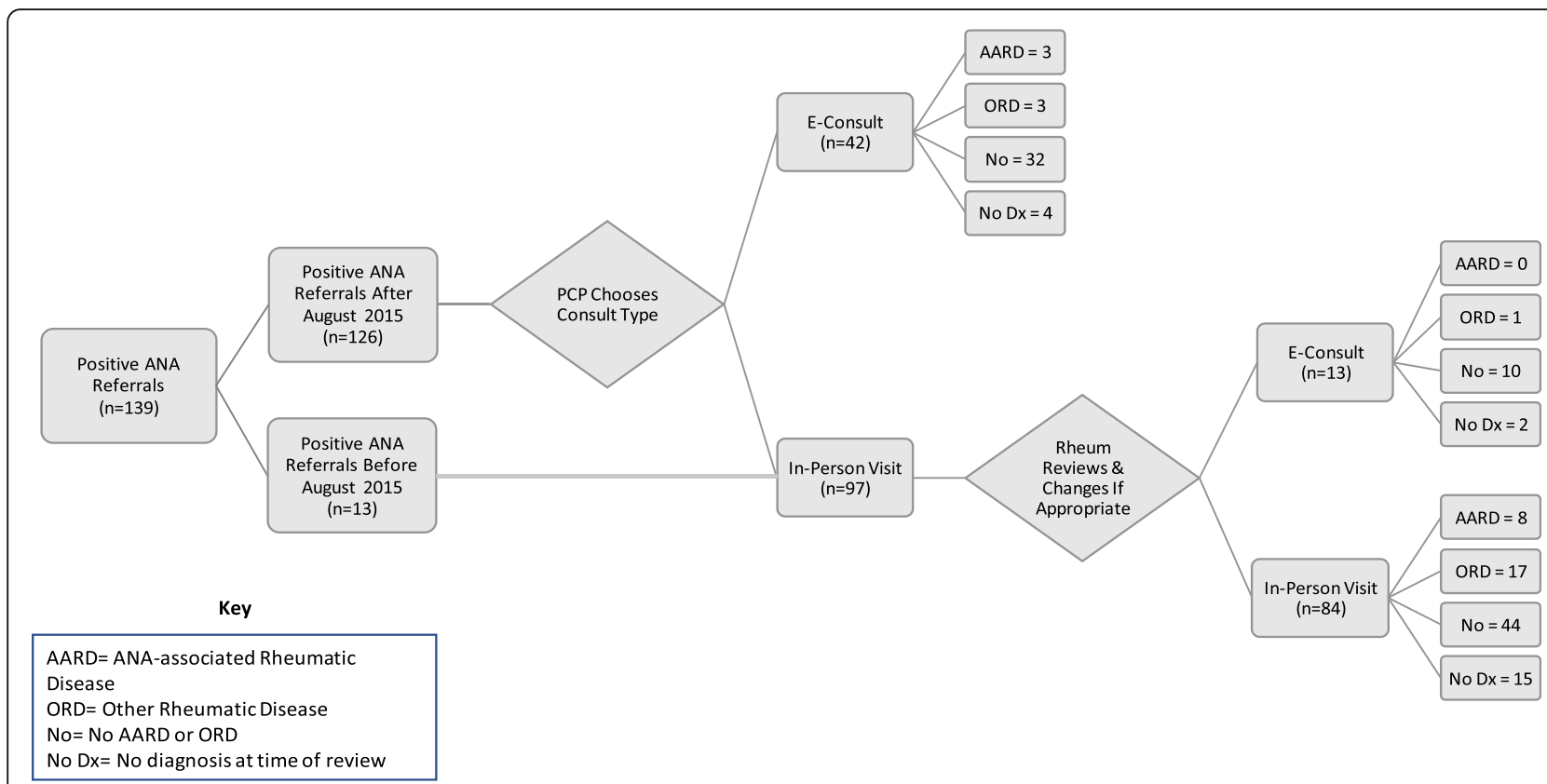

Fig. 1 The journey of positive ANA referrals through the referral system and the final diagnosis category. Primary care providers (PCP) choose the initial consult method (E-consult or in-person visit). A rheumatologist reviews all consults before appointments are scheduled and can change clinic visits to E-consults (or vice versa) if their chart review deems this appropriate. No in-person visits were changed to E-consults during our project

In our study, $76 \%$ of positive ANA referrals did not have further in-person follow-up with rheumatology after the initial E-consult. A prior study in rheumatology showed that $38 \%$ of E-consults avoided face-toface visits [2]. Focusing exclusively on one type of consult: the positive ANA, may explain our higher rate of evaluation by E-consults alone. Also, our patient population of predominantly older white men, are not a high-risk group for AARD and likely had an ANA ordered without clinical signs and symptoms of
AARD. Anti-DFS70 antibodies are commonly positive in healthy individuals and are not likely indicative of AARD, even at high titers [19-21]. Incorporating anti-DFS70 antibodies into the evaluation of positive ANA E-consults could be a potential way to avoid unnecessary testing [19] and give reassurance that a patient will not likely develop an AARD over time [20]. The average rheumatology response time to Econsult in our study was similar to prior studies using asynchronous telehealth modalities $[2,10]$.

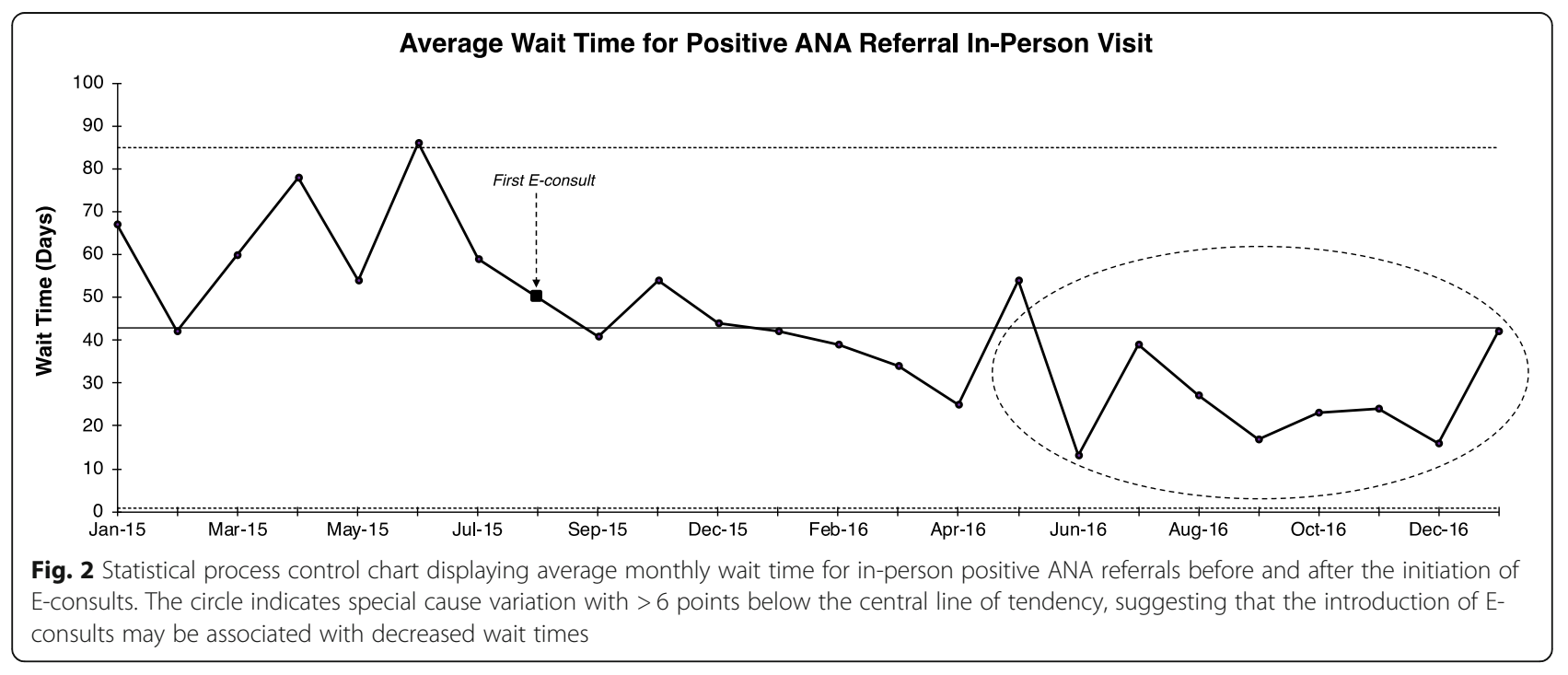


Table 2 Resource utilization between E-consults and in-person visits for different diagnosis groups. Overall, more lab tests were ordered per person by E-consults, but this was not statistically significant. In-person visits ordered more imaging studies. Labs and imaging studies are reported as a mean per person with the range listed in parentheses

\begin{tabular}{llll}
\hline Resource Utilization of Positive ANA Referrals & & \\
\hline & $\begin{array}{l}\text { In-person } \\
\text { Visits } \\
n=84\end{array}$ & $\begin{array}{l}\mathrm{E}- \\
\text { consults } \\
n=55\end{array}$ & $\begin{array}{l}\boldsymbol{p} \text { - } \\
\text { value }\end{array}$ \\
& $3.2(0-9)$ & $3.9(0-10)$ & 0.11 \\
& $1.1(0-9)$ & $0.1(0-2)$ & 0.0003 \\
\hline Labs (per person) & & & \\
Imaging Studies (per person) & 8 & 3 & \\
Final Diagnoses & 3.1 & 5.7 & 0.11 \\
AARD & 1.8 & 0 & 0.37 \\
$\quad$ Labs & 17 & 4 & \\
$\quad$ Imaging Studies & 3.5 & 1.8 & 0.23 \\
ORD & 1.8 & 0 & 0.15 \\
$\quad$ Labs & 44 & 42 & \\
$\quad$ Imaging Studies & 3.1 & 3.9 & 0.18 \\
No Rheumatic Disease & 0.6 & 0.1 & 0.01 \\
$\quad$ Labs & 15 & 6 & \\
$\quad$ Imaging Studies & & & \\
No Diagnosis (at time of \\
review)
\end{tabular}

AARD ANA-associated rheumatic disease, ORD Other rheumatic disease

Telehealth has been identified as a viable option to continue providing timely care during the current COVID-19 pandemic [1] and many providers have quickly adapted to using telemedicine modalities including E-consults. Not only are E-consults a way to continue care during a quarantine period, but as our results indicate, they are also a considerable way to decrease in-person rheumatology wait times.

Our study is limited by its small scope and short duration. We do not have long-term follow-up data to see if resolved E-consults eventually needed an in-person visit or developed an AARD. We also do not have information about the decision-making process or criteria that was used when reviewing an E-consult, future projects could consider evaluating this to improve or standardize the E-consult process. Also, final diagnoses were based on evaluating the rheumatologist's clinic note and there was no confirmation by a second rheumatologist of the diagnosis. This study may not be generalizable for health care systems that receive referrals from outside sources and those that do not share an EHR or web-based platform with their referring providers. We also focused on a subset of referrals and do not know if wait times for all types of referrals improved.

\section{Conclusions}

With the ongoing COVID-19 pandemic and surge in telehealth practices, E-consults will likely continue to be incorporated into specialty care, including rheumatology. Our study shows that E-consults are an effective way to address positive ANA referrals without a significant increase in resource use, so patients that require in-person evaluation can be seen in a timely manner. Rheumatologists considering continuing or starting telehealth initiatives may consider focusing on positive ANA referrals to improve wait times for in-person visits.

\section{Abbreviations \\ ANA: Anti-nuclear antibody; E-consult: Electronic consult; EHR: Electronic health record; AARD: ANA-associated rheumatic disease; Anti-CCP: Anti-cyclic citrullinated peptide; SS-A: Anti-Ro; SS-B: Anti-La; dsDNA: Anti-double stranded DNA; C3: Complement C3; C4: Complement C4; CT: Computed tomography; MRI: Magnetic resonance imaging; ORD: Other rheumatic disease; SLE: Systemic lupus erythematosus; MCTD: Mixed connective tissue disease; PM: Polymyositis; DM: Dermatomyositis; UCTD: Undifferentiated connective tissue disease; IRB: Institutional Review Board}

\section{Acknowledgments \\ Dr. Joan Appleyard.}

\section{Authors' contributions}

VP-Contributed to the conception of the work, acquisition of data, data analysis, drafted the work and approved submitted version. DS -

Contributed to the conception of the work, substantially revised it, and approved submitted version. $\mathrm{MH}$ - Contributed to the conception of the work, interpretation of the data, substantially revised it, and approved submitted version.

\section{Author information}

The views expressed in this article are those of the authors and do not necessarily reflect the position or policy of the Department of Veterans Affairs, the United States government, or Baylor College of Medicine.

\section{Funding}

This work is supported in part by the Department of Veterans Affairs, Veterans Health Administration, Office of Research and Development, Houston Health Services Research and Development Center for Innovations in Quality, Effectiveness, and Safety (IQuESt) grant (CIN 13-413) at the Michael E. DeBakey VA Medical Center, Houston, TX. This work is also supported by the American Heart Association Grant 17MCPRP33390011 (Horstman).

\section{Availability of data and materials}

The datasets generated during and/or analyzed during the current study are not publicly available due to this work being designated as quality improvement but are available from the corresponding author on reasonable request.

\section{Ethics approval and consent to participate}

This study was reviewed by the Institutional Review Board (IRB) and local Research and Development Committee and designated a quality improvement project.

\section{Consent for publication}

N/A

\section{Competing interests}

None.

\section{Author details}

${ }^{1}$ Division of Rheumatology, Department of Medicine, University of Texas at Austin, Dell Medical School, 1601 Trinity St., Bldg B, Stop Z0900, Austin, TX 78712, USA. ${ }^{2}$ Section of General Internal Medicine, Department of Medicine, Baylor College of Medicine, Houston, TX, USA. ${ }^{3}$ VA HSR\&D, Center for Innovations in Quality, Effectiveness and Safety, Michael E. DeBakey VA Medical Center, Houston, TX 77030, USA. ${ }^{4}$ Section of Health Services Research, Department of Medicine, Baylor College of Medicine, Houston, TX, 
USA. ${ }^{5}$ VA Quality Scholars Coordinating Center, IQuESt, Michael E. DeBakey VA Medical Center, Houston, TX, USA.

Received: 12 May 2020 Accepted: 29 July 2020

Published online: 15 October 2020

\section{References}

1. Wosik J, et al. Telehealth transformation: COVID-19 and the rise of virtual care. J Am Med Inform Assoc. 2020;27(6):957-62.

2. Rostom $K$, et al. Improving access to rheumatologists: use and benefits of an electronic consultation service. J Rheumatol. 2018;45(1):137-40.

3. Vimalananda VG, et al. Electronic consultations (e-consults) to improve access to specialty care: a systematic review and narrative synthesis. J Telemed Telecare. 2015;21(6):323-30.

4. Winchester DE, et al. Electronic consults for improving specialty care access for veterans. Am J Manag Care. 2019;25(5):250-3.

5. Rezaian MM, et al. Rheumatology care using telemedicine. Telemed J E Health. 2020;26(3):335-40.

6. Wood PR, Caplan L. Outcomes, satisfaction, and costs of a rheumatology telemedicine program: a longitudinal evaluation. J Clin Rheumatol. 2019; 25(1):41-4.

7. Devadula S, et al. Tele-rheumatology to regional hospital outpatient clinics: patient perspectives on a new model of care. Telemed J E Health. 2020; 26(7):912-9.

8. Kulcsar Z, et al. Telerheumatology: a technology appropriate for virtually all. Semin Arthritis Rheum. 2016:46(3):380-5.

9. Taylor-Gjevre R, et al. Addressing rural and remote access disparities for patients with inflammatory arthritis through video-conferencing and innovative interprofessional care models. Musculoskeletal Care. 2018;16(1):90-5.

10. Scheibe $M M$, et al. Efficiency gains for rheumatology consultation using a novel electronic referral system in a safety-net health setting. Arthritis Care Res (Hoboken). 2015;67(8):1158-63.

11. Abeles AM, Abeles M. The clinical utility of a positive antinuclear antibody test result. Am J Med. 2013;126(4):342-8.

12. Slater CA, Davis RB, Robert H. Shmerling, Antinuclear Antibody Testing: A Study of Clinical Utility. Arch Intern Med. 1996;156:1421-5.

13. Soto $M E$, et al. Predictive value of antinuclear antibodies in autoimmune diseases classified by clinical criteria: analytical study in a specialized health institute, one year follow-up. Results Immunol. 2015;5:13-22.

14. Satoh $\mathrm{M}$, et al. Prevalence and sociodemographic correlates of antinuclear antibodies in the United States. Arthritis Rheum. 2012;64(7):2319-27.

15. Solomon $\mathrm{DH}$, et al. Evidence-based guidelines for the use of immunologic tests: antinuclear antibody testing. Arthritis Rheum. 2002;47(4):434-44.

16. Dinse $G E$, et al. Increasing prevalence of antinuclear antibodies in the United States. Arthritis Rheumatol. 2020;72(6):1026-35.

17. Battafarano DF, et al. 2015 American College of Rheumatology Workforce Study: supply and demand projections of adult rheumatology workforce, 2015-2030. Arthritis Care Res (Hoboken). 2018;70(4):617-26.

18. Provost, L.P. and S.K. Murray, The health care data guide : learning from data for improvement. 1st ed. 2011, San Francisco: Jossey-Bass. Xxviii, 445 p.

19. Gundin S, et al. Measurement of anti-DFS70 antibodies in patients with ANA-associated autoimmune rheumatic diseases suspicion is cost-effective. Auto Immun Highlights. 2016;7(1):10.

20. Mariz HA, et al. Pattern on the antinuclear antibody-HEp-2 test is a critical parameter for discriminating antinuclear antibody-positive healthy individuals and patients with autoimmune rheumatic diseases. Arthritis Rheum. 2011;63(1):191-200

21. Tonutti $E$, et al. The ANA-reflex test as a model for improving clinical appropriateness in autoimmune diagnostics. Auto Immun Highlights. 2016;7(1):9.

\section{Publisher's Note}

Springer Nature remains neutral with regard to jurisdictional claims in published maps and institutional affiliations.

Ready to submit your research? Choose BMC and benefit from:

- fast, convenient online submission

- thorough peer review by experienced researchers in your field

- rapid publication on acceptance

- support for research data, including large and complex data types

- gold Open Access which fosters wider collaboration and increased citations

- maximum visibility for your research: over $100 \mathrm{M}$ website views per year

At $\mathrm{BMC}$, research is always in progress.

Learn more biomedcentral.com/submissions 